\title{
Global and Local Processing in Adult Humans (Homo sapiens), 5-Year-Old Children (Homo sapiens), and Adult Cotton-Top Tamarins (Saguinus oedipus)
}

\author{
Julie J. Neiworth, Amy J. Gleichman, Anne S. Olinick, and Kristen E. Lamp \\ Carleton College
}

\begin{abstract}
This study compared adults (Homo sapiens), young children (Homo sapiens), and adult tamarins (Saguinus oedipus) while they discriminated global and local properties of stimuli. Subjects were trained to discriminate a circle made of circle elements from a square made of square elements and were tested with circles made of squares and squares made of circles. Adult humans showed a global bias in testing that was unaffected by the density of the elements in the stimuli. Children showed a global bias with dense displays but discriminated by both local and global properties with sparse displays. Adult tamarins' biases matched those of the children. The striking similarity between the perceptual processing of adult monkeys and humans diagnosed with autism and the difference between this and normatively developing human perception is discussed.
\end{abstract}

Keywords: global precedence, local bias, perception, discrimination, cotton-top tamarins

A critical early process of visual cognition is the perceptual parsing of the visual input or, more specifically, the cognitive work of grouping features for object identification. This seems to happen in humans via two broad categories or principles: global property assessment, by which the viewer attends to overall shape despite local feature differences, and gestalt principles, by which the viewer connects features into groupings (like proximity, similarity, and closure) to define shapes. In the simplest of terms, global assessment involves object identification by whole contour and is not affected by local element change, whereas gestalt assessment involves object identification by interelement relationships and thus is critically affected by local element change. Because of this difference, global processing has been described as the processing of place relationships (Kimchi, 1990, 1992), whereas gestalt processing has been described as the processing of nature relationships wherein it is necessary to assess operations both within and between groups of elements to identify objects (Quinlan \& Wilton, 1998).

Adult humans process visual stimuli by gestalt principles and by global properties first. Various researchers have demonstrated a global precedence effect (Navon, 1977, 1981) by which human adults respond more quickly to the global properties (i.e., the shape

Julie J. Neiworth, Amy J. Gleichman, Anne S. Olinick, and Kristen E. Lamp, Department of Psychology, Carleton College.

Amy J. Gleichman is now a graduate student in neuroscience at University of Pennsylvania. Anne S. Olinick is now a researcher at ETR Associates, San Francisco, CA. Kristen E. Lamp is now a graduate student at Loyola University.

This research was supported in part by National Institute of Mental Health Grant 1 R15 MH071232-01A2 to Julie J. Neiworth, in part by Carleton College, and in part by the Howard Hughes Medical Institute.

Correspondence concerning this article should be addressed to Julie J. Neiworth, Department of Psychology, Carleton College, Northfield, MN 55057. E-mail: jneiwort@carleton.edu and overall contour) of figures constructed of smaller stimuli (the local property) and notice the elements of construction only later in the process. Global assessment and gestalt principles share common characteristics in human perceptual processing; for example, both seem to occur primarily in the right hemisphere in humans (van Kleek \& Kosslyn, 1989), and both occur temporally early in the processing of percepts (Triesman \& Peterson, 1984). By these characteristics, one may assume that these processes are specialized adaptations in humans and may have evolved as hardwired modules of the human perceptual system. Evidence from developmental studies has suggested that the bias emerges with development and is constrained by the stimulus context. Cassia, Simion, Milani, and Umitla (2002) found that newborn infants look longer at, and thus attend to, changes in both local features and global features, and Ghim and Eimas (1988) found similar results in 3 -month-olds. In one experiment in each of these multiple experiment studies, there was a global advantage demonstrated, but only in a particular task in which two novel stimuli with conflicting global-local changes were shown as test items. This suggests that human perception may be tuned to process global properties through experience and that the global bias emerges when assessing novelty in stimuli that change both globally and locally. Recent research has indicated that perceptual groupings based on form similarity are not demonstrated by infants until 6 or 7 months of age, suggesting that gestalt grouping principles also emerge over time and with experience (Quinn, Bhatt, Brush, Grimes, \& Sharpnack, 2002).

Global and gestalt processing are clearly tuned with experience in humans to show a particular bias. It is intriguing to ask whether other primates that are not human show a particular bias as adults. Human and nonhuman primates' visual systems seem quite similar in processing at the sensory level (De Valois \& Jacobs, 1968; Fobes \& King, 1982), but perhaps changes in perceiving occur at a later perceptual or cognitive level. Some researchers have suggested that the global precedence effect emerged in recent primate 
evolution and that it splits the perceptual processing styles of monkeys from those of apes, including humans (Fagot, Tomonaga, \& Deruelle, 2001; Hopkins \& Washburn, 2002). Global processing biases allow primates to generalize across objects that differ in many local details and thus would allow broad superordinate categories to be formed and labeled, like a type of animal or tree as opposed to other fauna or flora. Local processing would yield discriminations based on internal changes of features, like color or texture, and would be relevant to discrimination of ripe foods or the identity of individuals. There are obvious advantages to discriminating on the basis of local detail and on the basis of global change, and it is likely the case that many species of animals can discriminate on the basis of both sets of cues (e.g., for pigeons see Fremouw, Herbranson, \& Shimp, 2002). However, the kinds of biases different species of animals show when confronted with similar stimuli indicate the basic perceptual differences in how they naturally see objects in the world. It is possible that a marker for evolutionary differences is how the brain naturally processes features of objects, with certain hierarchical organizations of input leading to different levels of cognitions. In this way, the organization of perceptual input may define important cognitive differences in the thinking of apes as opposed to monkeys.

Studies of chimpanzees have demonstrated global precedence, as evidenced by their attending to the outer contour of forms (Tomonaga \& Matsuzawa, 1992), by their learning to respond first to the outer contour (Fujita \& Matsuzawa, 1990), or by their processing of the overall shape of forms better than the local elements by which the forms were constructed (Hopkins \& Washburn, 2002). It is interesting to note that chimpanzees sometimes fail to show a global precedence effect though. Fagot and Tomonaga (1999) tested chimpanzees in a compound visual search task and found that humans but not chimpanzees showed a global stimulus processing advantage. In this study, chimpanzees attended to both local and global levels and showed an advantage for processing at a local level when stimuli were presented in a sparse display (with an interelement distance larger than $0.4 \mathrm{~cm}$ ). In the Hopkins and Washburn (2002) study that showed a global precedence effect in chimpanzees, the interelement distance was 1.0 $\mathrm{mm}$.

A number of studies have suggested that many species of New World and Old World monkeys show a local bias in that researchers suggested that monkeys process first or more accurately the local features of stimuli. Curiously, the effect is often expressed not by a local bias but by a lack of global precedence effect. For example, a lack of global precedence effect was reported in rhesus monkeys in a sequential matching-to-sample task (Hopkins \& Washburn, 2002). By response accuracy, the rhesus monkeys attended to both local and global changes to match stimuli that were presented. Tanaka and Fujita (2000) found mixed results in terms of response accuracy by 2 rhesus monkeys to global and local changes because 1 monkey showed a global advantage in accuracy whereas the other seemed equivalently accurate in local and global tasks. Fagot and Deruelle (1997) tested baboons and humans in a split-screen matching-to-sample task and showed a local processing preference in baboons, later replicating the global precedence effect with the same stimuli in humans. The experimenters found that reducing the interelement distance had some effect on overcoming the local precedence effect in baboons (Fagot et al., 2001). Spinozzi, De Lillo, and Truppa (2003) also found a local processing bias in capuchin monkeys in a simultaneous matching-to-sample task by using displays with interelement distances of $0.6 \mathrm{~cm}$, but this local bias was diminished, and equivalent processing of both global and local displays occurred with dense displays with interelement distances of approximately $0.4 \mathrm{~cm}$. The performance of the monkeys with dense displays was very similar to the matching accuracies of 3- and 4.5-year-old children in the same task (De Lillo, Spinozzi, Truppa, \& Naylor, 2005). Unlike the monkeys, though, the children showed equivalently high accuracies to local or global changes regardless of the density of the stimulus presentation. It is important to note that the children in this study were in two sequential experiments in which they were first exposed to medium displays and then exposed to dense and sparse displays. The children matched correctly all trials, but this might be due to the extensive exposure they received. Dukette and Stiles (1996) reported that 4-year-old children with limited exposure to the training stimuli show a local bias with sparse element displays but not with dense element displays.

In humans, babies often show attention differences at the local and global levels. Young children have shown a heightened sensitivity toward local changes with sparse displays (Dukette \& Stiles, 1996) but typically have shown sensitivities toward both local and global properties with dense displays (De Lillo et al., 2005; Dukette \& Stiles, 1996). Older children and adults have shown a global precedence effect that is unaffected by stimulus display densities (Dukette \& Stiles, 1996, 2001), and so the perceptual processing of stimuli by adult humans is quite different from that which occurs in early cognitive development. Do other adult primates show a bias toward global assessment? The data suggest the answer is most often no. Some studies reported a performance advantage in monkeys to local changes in the stimulus when sparse element displays were used, suggesting that adult monkeys' processing matches young human children's toward global or local biases based on stimulus conditions. It is important to note here that whereas young children's processing obviously changes developmentally from a focus induced by stimulus conditions to a focus that is predominantly global, the monkeys show a stimulus-induced processing style as adults and thus show a different endpoint to their development and experience. Even more important, the monkeys' processing style is inferred from only a low number of studies, most of which do not compare the results of adults and children with adult monkeys. It is important to compare directly the discrimination performances of adult humans, young children, and monkeys in a task using sparse and dense stimuli to definitively demonstrate whether adult monkeys' abilities match those of young children and to determine whether either of these two groups matches the processing style of adult humans.

In our study, a total of 35 college students, twelve 5-year-old children, and 8 adult cotton-top tamarins were divided into two groups so that approximately half were in a "few elements" condition ( $n=17, n=6$, and $n=4$, respectively) and half were in a "many elements" condition $(n=18, n=6$, and $n=4$, respectively). All subjects participated in a training discrimination in which they had to respond differentially to circles made out of small blue circle elements $(\mathrm{Cc})$ and squares made out of small blue square elements (Ss). Two different densities of the smaller elements were used to create the "few" and "many" conditions. In the few condition, there were 8 elements that made up the global shape. In the many condition, there were 16 elements used to make 
up the global shape. For humans, the training task was a category discrimination in which they learned to hit one letter on a computer keyboard when they saw a $\mathrm{Cc}$ and a different letter on the keyboard when they saw an Ss. Humans saw only one stimulus at a time but had to use memory of salient perceptual cues to determine which particular stimulus required a particular response to acquire a reward (in the form of a happy face). A computer task was used for humans to collect reaction times as well as accuracy, for it was unclear a priori whether accuracy could vary across the types of stimuli (few vs. many), whereas time to respond might.

For monkeys, the training task was a go/no-go discrimination in which they were required to respond to a card with a $\mathrm{Cc}$ and not an Ss to acquire rewards. The monkey task was slightly different than the human task in that monkeys were presented with two cards and were required to select one to complete a trial. Still, the cognitive work is similar in that the monkeys had to use memory for which particular perceptual cues (circle shape or circle elements) were associated with a response to acquire rewards (in the form of cereal treats). It was found in prior operant work that the monkeys could not make discrete responses toward a computer screen to select a stimulus because of the physical constraints imposed by large paws, nor could they readily learn to push particular buttons to indicate a choice and, in fact, had great difficulty associating an arbitrary response on a manipulandum (like a lever or keyboard) with events. The monkeys readily selected items, such as cups and cards, and so a go/no-go task seemed preferable in that the exact stimuli could be used with the same response requirements in a slightly different format.

Subsequent to the training task, the test measured the kind of response emitted to stimuli that presented conflicting properties, for example a circle shape ( $\mathrm{S}+$ for global property) made of square elements ( $\mathrm{S}-$ for local property) or a square shape $(\mathrm{S}-$ for global property) made of circle elements $(\mathrm{S}+$ for local property). In humans, a categorical response on the keyboard would indicate which property they assessed predominantly in the new stimulus, global or local. In the monkeys, accuracy to select the novel card as an $\mathrm{S}+$ or an $\mathrm{S}-$ was assessed to determine to which property, global or local, they showed a bias. Selecting circle shapes regardless of the elements used to construct them would indicate a global bias, whereas selecting circle elements regardless of the shape they composed would indicate a local bias in monkeys.

\section{Method}

\section{Subjects}

Human children (Homo sapiens). There were 13 children between 55 months and 62 months of age who participated. Of the subjects, 1 was eliminated because he failed to reach the $80 \%$ correct criterion in the training phase of the study. The subjects were solicited from Longfellow School, which housed 10 different kindergarten classes in Northfield, Minnesota. The children were scheduled for a 40-min session in the evening in a laboratory at Carleton College, and a parent or guardian was present who gave consent by signing a form. Subjects were rewarded with stickers, a certificate, and a t-shirt for volunteering. They were randomly placed into the few or many condition by a flip of the coin, with the additional constraint of counterbalancing the number of subjects between the two conditions.

Human adults (Homo sapiens). A total of 35 college students were solicited for participation from various introductory courses at Carleton College. All subjects were between 18 and 22 years of age, and all signed a consent form before participating in a 30-min session scheduled at an evening time in the laboratory. The subjects were placed in either the few or the many condition by a flip of a coin, with the constraints of counterbalancing number across the two conditions and making the genders equivalent within each condition. There were 17 students placed in the few condition and 18 placed in the many condition.

Adult cotton-top tamarins (Saguinus oedipus). A total of 8 adult cotton-top tamarins, 4 female tamarins (Fozzy, Encore, Ophelia, and Olympia) and 4 male tamarins (Mac, Zhivago, Rolo, and Willow), were placed in one of two groups (few or many) for the study. There were 2 of each sex placed in the few group and 2 of each sex placed in the many group. All subjects had been monkey-family reared in laboratory settings and had been socially housed in pairs in five different $0.85 \times 1.50 \times$ $2.30 \mathrm{~m}$ cages, with the cages visually separated by opaque sheets. The subjects were on a 12-hr light-dark cycle and had free access to water. All animals were maintained on a complete diet consisting of a yogurt and applesauce breakfast, a lunch of Zupreem Marmoset chow, Mazuri New World Monkey dry chow, fruits and vegetables, and a protein snack (e.g., eggs, hamburger, mealworms) daily. Subjects had been exposed to mirrors and to digitized pictures of themselves (Neiworth, Anders, \& Parsons, 2001), to digitized pictures of other animals (Neiworth, Parsons, \& Hassett, 2004), and to hidden treats in cups (Neiworth, Burman, Basile, \& Lickteig, 2002; Neiworth et al., 2003). None of them had been exposed to graphic stimuli on cards before this study, and none had been trained to select one of two cards in a response task before.

\section{Materials}

The stimuli in the training phase were $\mathrm{Cc}$ and Ss. The circle elements were $4.0 \mathrm{~mm}$ in diameter, and the square elements were $4.0 \mathrm{~mm} \times 4.0 \mathrm{~mm}$. The global circle shapes were $5.5 \mathrm{~cm}$ in diameter, whereas the global square shapes were $4.1 \mathrm{~cm} \times 4.1 \mathrm{~cm}$. Examples of each are shown in Figure 1.

Two different densities of the smaller elements were used to create the few and many conditions. In the few condition, 8 elements made up the global shape, with an approximate interelement distance of $1.4 \mathrm{~cm}$. In the many condition, 16 elements were used to make up the global shape, with an interelement distance of approximately $0.4 \mathrm{~cm}$. For humans, these shapes were presented, via PsyScope 1.2.5 (Cohen, MacWhinney, Flatt, \& Provost, 1993) on a Power Macintosh G4 computer, at any one of five different locations on a white background projected on a monitor measuring $44.0 \mathrm{~cm}$ diagonally. The stimulus moved across trials to different locations to engage the attention of the human subjects. For tamarins, these stimuli were printed at the center of $7.6 \mathrm{~cm} \times 12.7 \mathrm{~cm}$ laminated white cards.

For the test, four new stimuli were constructed with the two density arrays and a mixing of the same global shapes and same elements. They included a circle made of squares (Cs) and a square made out of circles $(\mathrm{Sc})$. These were constructed with few elements, or 8 elements, and with many elements, or 16 elements. Examples are provided in Figure 1.

\section{Apparatus}

The apparatus used for human adults and children was an Apple Power Macintosh G4 computer on which the stimuli were presented on the monitor, and responses were recorded from its keyboard. The keyboard's keys were covered by a cardboard sheet, with squares cut out to expose only the $Z$ and the slash keys. The program PsyScope (Version 1.2.5) was used to design the trials, present the stimuli, and collect responses, including keyboard responses and reaction times.

The apparatus used for tamarins was a white projector cart measuring $1.17 \mathrm{~m}$ in height with a top shelf measuring $40 \mathrm{~cm} \times 50 \mathrm{~cm}$. On any trial in training or testing, two cards measuring $7.6 \mathrm{~cm} \times 12.7 \mathrm{~cm}$ were placed $20 \mathrm{~cm}$ from the front (subject side) of the cart and $10 \mathrm{~cm}$ from the sides of the cart, with a separation of $20-30 \mathrm{~cm}$ from each other. 


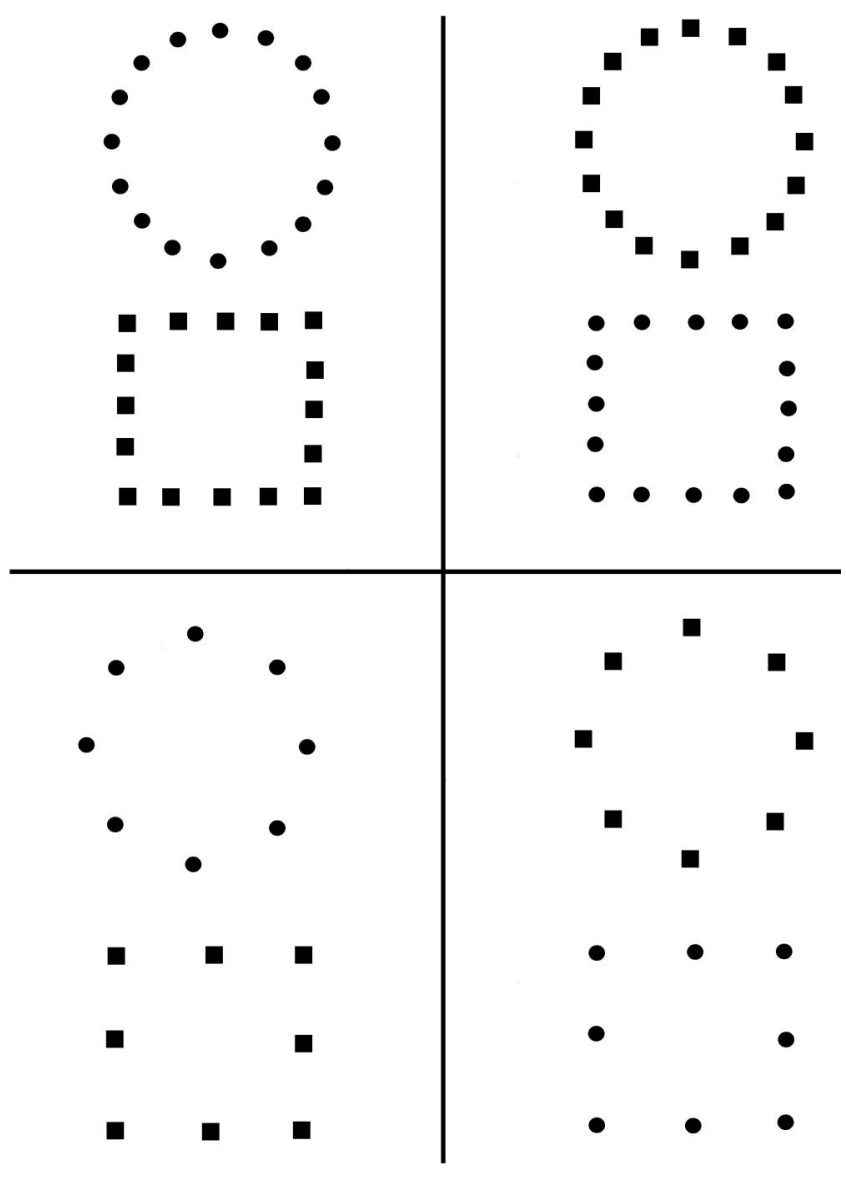

Figure 1. The left panel shows the training stimuli, with the $\mathrm{S}+$, the circle made of circles, and the $\mathrm{S}-$, the square made of squares. The right panel shows the test conflict stimuli, which combine $\mathrm{S}+$ and $\mathrm{S}-$ properties. The top panel shows the stimuli used in the "many" condition; the bottom panel shows the stimuli in the "few" condition.

\section{Procedure}

Training and testing children. After interacting with parent and child, obtaining consent from the parent, and seating the child in front of the computer, the following instructions were read to each subject:

I'm going to show you two different objects on this screen. See the $Z$ and the slash keys? You should put your fingers on them. One key goes with one object, and the other key goes with the other object. I can't tell you which one goes with which, but the computer will smile at you if you figure it out. It will also show you a frown if the key does not go with the object you see. Try to get as many smiles as you can.

Subjects saw stimuli only from their designated condition, so subjects in the few condition saw stimuli constructed of 8 elements both in training and in testing, and subjects in the many condition saw stimuli constructed of 16 elements both in training and in testing. The training session comprised twenty 60-s trials that presented $\mathrm{Cc}$ and Ss 10 times each, pseudorandomly mixed with the constraints that no more than three of one type could appear consecutively and all five monitor locations were used equivalently. At the beginning of each trial, a central fixation point asterisk was shown on the screen for $300 \mathrm{~ms}$. Then a stimulus, either a Cc or an Ss, was presented at any one of five locations on the screen: centered on the screen or in the upper left, upper right, lower left, or lower right quadrants of the screen. The stimulus remained on until a keyboard response was made or until $60 \mathrm{~s}$ elapsed. Responses to the $Z$ key were correct for the Cc, and responses to the slash key were correct for the Ss. If the subject responded correctly, a cartoon face smiling appeared in the center of the screen for $15 \mathrm{~s}$. If the subject responded incorrectly, a cartoon face frowning appeared for $15 \mathrm{~s}$. There was a varying intertrial interval between trials such that each subject could wait until he or she was ready to start the next trial. The next trial was initiated by the experimenter by hitting the space bar on the keyboard, and this was done when the child was looking at the screen again and had fingers on the two relevant keys on the keyboard. If no response was emitted within the 60-s trial, a blank screen followed and the experimenter started the next trial with a keyboard response when the child appeared ready to play.

If the subject reached a criterion of $80 \%$ accuracy within the first phase of training, he or she would proceed to testing. If accuracy was less than $80 \%$, another training session was initiated. If the subject did not reach criterion after the second session, the subject was given his or her rewards and the study ended without a test. Of the subjects, 1 was not tested, and the other 12 subjects learned the task and proceeded to testing.

The instructions during the test session were as follows:

Good work! Now I have one more game involving objects for you to play. This time the same two objects will appear, and the same two keys need to be touched to get smiley faces. But there are also some more objects mixed in here and I don't know the right answer to them so you won't see a smile or a frown to them. I just want you to tell me by hitting one of those two keys which key you think the object belongs to.

The test session comprised 32 trials, with 20 training trials and 12 test trials intermixed in a pseudorandom fashion with the constraint that no more than three trial types were repeated. The trials all progressed as before, with a fixation point for $300 \mathrm{~ms}$ followed by the stimulus for $60 \mathrm{~s}$. The 20 training trials were $\mathrm{Cc}$ and $\mathrm{Ss}$, presented twice at each of the five screen locations. The 12 test trials were $\mathrm{Cs}$ and $\mathrm{Sc}$, and each of these types was presented twice at each of three screen locations (center, upper left, and upper right; or center, lower left, and lower right). The same two keys, $Z$ and slash, were the response keys and were rewarded $(Z$ for the $C c$ and slash for the Ss) in the training trials. In test trials, no feedback was given to the subject for a response. The $Z$ key response was coded a local response for the stimulus Sc because it indicated that the subject categorized the shape made of circle elements as a circle type. Similarly, the slash key response was coded as a local response for the stimulus Cs. On the other hand, if the stimulus Cs generated a $Z$ key response, then that was coded a global response because the subject was categorizing on the basis of the global shape and not the elements of construction. Similarly, a slash key response to the Sc stimulus was coded as a global response. Response times and responses were recorded, and accuracy was calculated for the training trials at the end of each session.

Training and testing adult humans. Once the adults signed consent to participate and were seated in front of the computer screen, each subject was read instructions similar to those read to the children, with the exception that the adult subjects were instructed to press the space bar when they wanted to initiate the next trial. This meant that their intertrial intervals could vary, but the actual trial length remained the same $(60 \mathrm{~s})$. The adults were presented the same training and testing programs as the children with the same two response keys trained to categorize $\mathrm{Cc}$ (the $Z$ key) and Ss (the slash key). All subjects were tested in the same manner and with the same number of test trials and feedback as the children. The adults received cookies and $\$ 5$ as a reward for participating in the experiment, rather than stickers and a t-shirt. Reaction times and response choices were recorded. 
Training and testing adult monkeys. Daily training sessions presented stimuli with few elements or stimuli with many elements exclusively, on the basis of the subject's assignment to condition. Each session comprised twenty 60-s trials. For each trial, the subject's name was called to gain his or her attention and then two cards were placed on the cart inside the subject's home cage. One of the cards was a $\mathrm{Cc}$, whereas the other was an Ss. The circle stimulus was the $\mathrm{S}+$, and a response to it, defined as moving the card physically on the cart with a front paw, was rewarded with a piece of cereal (either Froot Loop or Frosted Cheerio). Once the two cards were placed on the cart, the experimenter started a stopwatch to time $60 \mathrm{~s}$ and stared at a fixation point straight ahead. She noted the choice of the tamarin by looking down only when the tamarin was making a choice and had moved a card. If the correct card was selected, the subject was handed a cereal. If the response was incorrect, the experimenter said "no" and removed both cards from the cart. An intertrial interval of $15 \mathrm{~s}$ separated the trials, and during that time the experimenter recorded the choice and selected cards for the next trial. If the subject did not make a response within $60 \mathrm{~s}$ of the start of a trial, the experimenter removed the cards and marked the trial as aborted. After a 15-s intertrial interval, the trial was repeated, and if the subject did not respond for three consecutive trials, the session was terminated.

The location of the $\mathrm{S}+$ was counterbalanced so that it occurred on the left and right sides equally often within any one session. A total of six different sequences of 20 trials were constructed and used pseudorandomly across days. Once subjects acquired $80 \%$ accuracy within a single session, they were tested.

There were five consecutive test sessions conducted, each with a different sequence of trials, and each with 10 training trials intermixed with 10 test trials. The training trials presented the $\mathrm{S}+$ and $\mathrm{S}-$, and correct responses were rewarded. There were 5 different test trial types, 4 of which compared a novel stimulus with a training stimulus, and 1 of which pitted two novel stimuli against each other. Any response to any card in the test trials was rewarded. The original $\mathrm{S}+$ card $(\mathrm{Cc})$ was paired with a novel circle (Cs) or with a novel square (Sc). The original $\mathrm{S}-$ was also paired with a novel square $(\mathrm{Sc})$ and a novel circle (Cs). In the critical conflict test trial, two novel stimuli were shown: a Cs was compared with an Sc. Both stimuli were equally novel, and both contained a negative property (squares) and a positive property (circles). If subjects processed the global property predominantly, then they would select the Cs card in the conflict test trials, and cards containing global circle shapes $(\mathrm{C})$ in the other examples. If the subjects processed the local property predominantly, then they would select the Sc card more often in the conflict test condition and cards containing circle elements in the other examples.

\section{Results}

First, it seemed that all subject groups learned the discrimination relatively easily. Of the 12 children who were tested, 1 child required two 20-trial training sessions to achieve $80 \%$ accuracy at categorizing the two objects. The other 11 children learned the discrimination in 1 training session. All 35 adult subjects learned the discrimination in 1 training session. On average, it took tamarins 9.62 sessions to achieve $80 \%$ correct performance in the discrimination task, with a range between 3 sessions and 17 sessions. The mean number of sessions to criterion for monkey subjects in the few condition was 12.75 sessions and the mean for subjects in the many condition was 6.50 sessions. By a MannWhitney $U$ test for independent groups with small samples (e.g., $n=4$ in each group), the difference in learning rates between the two groups of monkeys was a nonsignificant trend $(U=2, p=$ $.057)$ with power estimated from a parametric test of the same data at $0.36, F(1,6)=3.66, p=.10($ effect size $=0.38)$.

The important data to examine were the global response biases to test stimuli that showed conflicting properties. A fixed factor analysis of variance ${ }^{1}$ compared age (children vs. adults) and condition (few vs. many) on mean percentage of global responses per subject with both novel test stimuli (Cs and Sc). The main effect of age was significant, $F(1,43)=4.12, p=.048$ (effect size $=$ 0.09 , power $=0.51)$, and this was due to the adults responding with a stronger global bias $(M=86.19 \%)$ than the children $(M=$ $71.76 \%)$. The main effect of condition was also significant, $F(1$, $43)=7.40, p<.01($ effect size $=0.15$, power $=0.76)$, because of a stronger global bias in the many condition $(M=87.15 \%)$ than in the few condition $(M=77.67 \%)$. There was a significant interaction effect of Age $\times$ Condition, $F(1,43)=8.28, p<.01$ (effect size $=0.16$, power $=0.80$ ). Figure 2 depicts the interaction in terms of the mean percentage global responses by adult and young humans to the test stimuli. It is clear in this graph that the significant difference between children and adults and between the few and many conditions was induced by a significant loss of global bias by children in the few condition. Independent groups $t$ tests comparing children's mean global response scores in the few and many conditions verified a significantly stronger global bias in the many condition $(M=91.67 \%)$ than the few condition $(M=$ $51.85 \%), t(10)=-4.93, p<.01$. There was no significant difference between adults' global responses in the many and few conditions, $t(33)=0.14, p=.89$.

Because the number of monkey subjects in each condition was small $(n=4)$ and the task was not identical, a separate nonparametric Mann-Whitney $U$ test compared monkeys' global biases across the many and few conditions. A mean global response was calculated for each monkey on the basis of the percentage of trials on which the response choice was made toward the Cs elements as opposed to the Sc elements, when the two stimuli were paired together. The responses to these exact stimuli were analyzed for humans. The means for monkeys in the few and many conditions are presented in Figure 2, and the Mann-Whitney $U$ test revealed a significant difference in global response $(U=1, p=.03)$, with monkeys in the few condition showing equivalent choice between local and global cues $(M=52.50 \%)$ and the monkeys in the many condition showing a strong global bias to the novel stimuli $(M=$ $70.00 \%)$. Power of this effect generated from parametric comparisons of the same data is $0.64, F(1,6)=7.74, p=.03$ (effect size $=0.56$ ).

The monkeys in the few condition were either noting both local and global features and thus showing less bias toward a global response or, alternatively, resorting to responding at chance levels, thus generating $50 \%$ global response. One-sample $t$ tests were

\footnotetext{
${ }^{1}$ This analysis was conducted once it was determined from a Levene's test of equality of error variances that, whereas the two groups of humans varied in size, the data generated from the groups were similar in terms of distribution, $F(3,43)=0.80, p=.49$.
} 


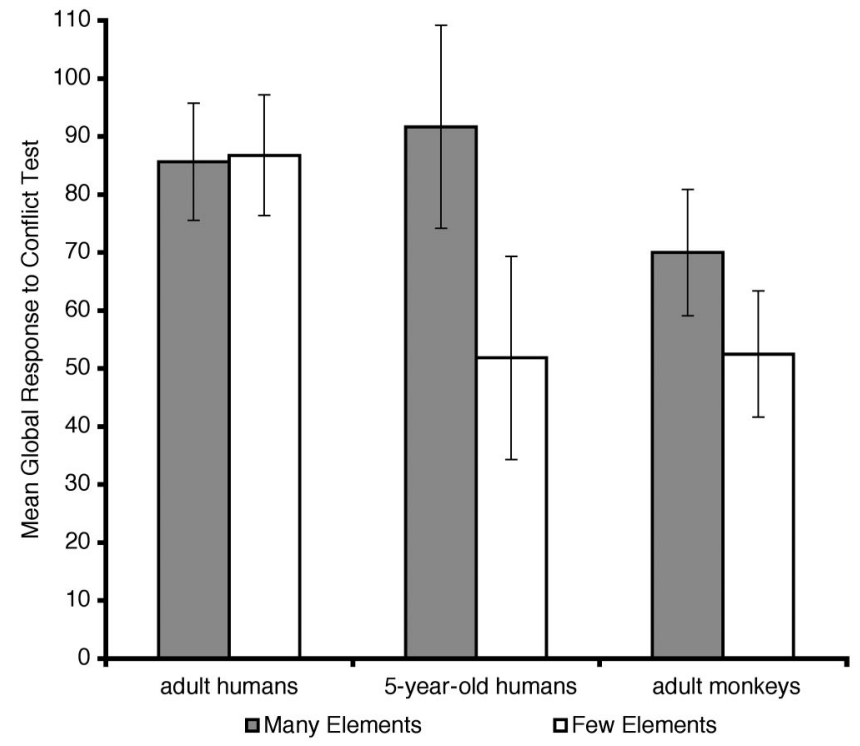

Figure 2. Mean global response to the conflict stimuli by adult humans, 5-year-old children, and adult tamarin subjects. Error bars are confidence intervals around the means.

conducted to compare the accuracies of these monkeys and the monkeys in the many condition with chance level responding, or $50 \%$ correct, to the training trials and the test trials. In the few test condition, the subjects' accuracy to the original discrimination was maintained at a level significantly above 50\% $(M=65.50 \%)$, $t(3)=4.84, p=.02$, but their global responding in the test trials showed equivalent responding to local and global cues $(M=$ $52.50 \%), t(3)=0.52, p=.64$. In the many condition in testing, the subjects' accuracy to the original discrimination was maintained significantly above chance level $(M=70.50 \%), t(3)=5.86, p=$ .01 , and their global response bias was significantly above a level of equivalence and toward global choices $(M=70.00 \%), t(3)=$ $4.90, p=.02$.

A final analysis examined the monkeys' global response bias toward the other novel trial types. A mean global response was calculated per subject as the percentage of times the subject selected the circle shape between the pairs in the test trials, regardless of the elements by which it was constructed. A MannWhitney $U$ test compared the mean global response of subjects in the many condition with the mean global response of subjects in the few condition, and it revealed a significant difference $(U=0$, $p=.014)$, indicating that the subjects in the many condition were significantly more biased toward global responding $(M=68.75 \%)$ than subjects were in the few condition $(M=48.12 \%)$.

\section{Discussion}

This study replicated the finding that adult humans are biased to note global properties of stimuli before local properties. In a task that required categorization via a keyboard response of graphic stimuli, the adult humans overwhelmingly responded to any global circle shape that appeared as though it belonged in the Cc category. They rarely put a different global shape, an Sc, in the Cc category. They seemed to ignore the local property of circle elements and led with global shape. This bias was unaffected by the density of the displays of local elements, for in both the few and the many elements condition, adult humans showed a strong global bias in their responses.

In contrast, 5-year-old children showed a global bias in their categorical assessment only if they were shown dense displays, as in the many elements condition. In this condition, the fact that the global shape was composed of 16 elements induced children to process the stimuli by global shape. This led them to show a strong global bias in categorizing $\mathrm{Cs}$ in the Cc category, and conversely, to not categorize Sc elements in the Cc category.

However, children of the same age with a similarly high level of accuracy before testing $(M=93.33 \%$ for children in the few condition) did not show a global bias in categorizing if they were originally presented shapes constructed of few elements. The children in this condition learned the discrimination very well, but their discrimination was based on both the global shape and the elements by which the shape was constructed. This led children to sometimes respond to a $\mathrm{Cs}$ as if it belonged to the Ss category, presumably because they shared local property features, and they sometimes put this novel stimulus into the Cc category because of its shared global property feature. It is important to note that the children in the few condition who showed this kind of equivalence judgment between local and global assessments were not poorer performers than the children in the many condition; children in both conditions showed very accurate performance to the training stimuli before the test $(95.00 \%$ and $93.33 \%$ accuracy, respectively), and they maintained this high level of performance during the test. They simply chose different ways to proceed when they saw the new stimuli that combined local and global features in novel ways. This difference in processing was induced by the change in the density of the displays. It is unlikely that visual acuity could cause the global bias in the many condition because in both conditions, the size of the elements remained the same, and the discriminations were acquired similarly. In sum, at this level of cognitive development, children seem capable of processing at the local and global levels equivalently but can become biased toward global assessments if features within the stimulus emphasize global properties (i.e., dense displays that emphasize the contour of the shape more). Dukette and Stiles $(1996,2001)$ found this same result in similarly aged children.

When New World monkeys were tested in a discrimination task very similar to the ones used with humans, their responses in testing revealed that they were biased toward global assessment if they had been trained with dense displays. When dense displays were used in training in the many condition, monkeys randomly placed in that condition continued to assess novel stimuli like Cs as if the global shape was the defining feature on which to discriminate. If sparse displays were used in training, as in the few condition, monkeys randomly placed in that condition learned the discrimination well but seemed to discriminate between both the local properties and the global properties. Thus, they sometimes chose the $\mathrm{Cs}$ as similar to the $\mathrm{S}+$, because of its global shape, and other times avoided it as though it were the $S-$, because of its square elements. The monkeys in the few condition did not show poorer performance in making the initial discrimination and maintained their original discrimination throughout testing, so this result is not due to the monkeys in the few condition transferring poorly to the test condition. There is no reason to conclude that 
tamarins would simply "guess" whenever they were presented novel cards in testing; in fact, individuals of the same species placed in the many elements condition did not resort to guessing when they saw the same novel cards in the test following the same kind of training. It is more parsimonious with the entire data set to conclude that the monkeys in the few condition, rather than guessing in novel trials, were actually attempting to discriminate, that they did so continually for the original $\mathrm{S}+$ and $\mathrm{S}-$, but that they also simply recognized the problems inherent in a stimulus containing both $\mathrm{S}+$ and $\mathrm{S}-$ properties and then categorized those problems as such.

Past published work has shown monkeys either to process global and local properties equivalently or to show a local processing bias. Our study suggests that monkeys can notice both the local and global properties of a stimulus and that the attentional allocation to each property is intricately tied to the stimulus itself. Monkeys attend to the local features of a stimulus especially when the stimulus features are spread out. Unlike adult humans, monkeys do not automatically favor perceiving the overall contour or place relationship. In fact, because the interelement distance seems the critical ingredient in monkeys' processing biases, the results suggest that monkeys may be biased toward perceiving the nature relationship in objects, or the interelement relationships. Why then did it take the monkeys almost twice as many sessions to learn the discrimination in the few condition? With sparse stimuli, an inability to inhibit the processing of local elements can localize what would normally be perceived as perceptually coherent, and this might make the global shape harder to perceive. Interpreted more broadly, this processing style would lead monkeys to learn more slowly all the features relevant in sparse arrays and show a conflict between attending to global and local features.

Young children show a similar failure to inhibit the processing of both local and global features when the stimuli they perceive are constructed of elements in a sparse array. We know that young children will develop the adult assessment style of global precedence at some point, most likely around age 6 , according to Dukette and Stiles (1996). Adult monkeys show a style of processing that favors both local and global processing and that is thus arrested at the level of young children.

The performance of monkeys as fully developed organisms seems more comparable with the perceptual processing of humans with autism. A critical finding that has emerged in the study of people with autism is that they note letters in Navon-style figures at both the global and local levels equivalently (Brosnan, Scott, Fox, \& Pye, 2004), and they show marked deficits in using gestalt principles as compared with delayed learning control groups. These outcomes suggest that individuals with autism cannot process higher level units (i.e., global properties) before lower level units (i.e., local properties), and they are less capable of perceiving interelement relationships as part of a global structure. There are some marked advantages that people with autism show that suggest a lack of hierarchy in their processing; for example, people with autism display an abnormal proficiency on tasks such as copying impossible figures (Mottron, Belleville, \& Menard, 1999) and finding embedded figures (Joliffe \& Baron-Cohen, 1997; Shah \& Frith, 1983). By not organizing in favor of global precedence, they find it easier to detect local details to find hidden objects and focus on local, albeit impossible, features of a stimulus.
It is possible that monkeys perceive stimuli in ways very similar to humans with autism. Young children also showed in this study a lack of hierarchical processing to certain stimuli, but because their visual-cognitive system will develop to show global bias and hierarchical processing after age 6 , the abnormality demonstrated by young children is not a good model of the fixed abnormality existing in autism-diagnosed people. Because monkeys are showing this problem as adults, the processing conflict for them seems fixed. Speculative claims about the source of this problem in autistic people include a lack of connectivity between brain areas that would normally allow for hierarchical pattern processing to develop or a lack of temporal binding between firing patterns that would normally produce templates or global representations of objects (Brock, Brown, Boucher, \& Rippon, 2002). More research testing monkeys' ability to assess global and local properties, the malleability of assessments based on interelement distances, and monkeys' assessment of gestalt groupings is needed to verify the similarity in perceptual processing between people with autism and monkey subjects. If supported, an animal model of autism could emerge that would help us to identify the neural mechanisms responsible for the perceptual deficits found in autism. An animal model could produce benefits in terms of developing tests for earlier diagnosis on the basis of perceptual reactions to stimuli. An animal model would allow for development of different treatment programs that may allow neural training or enhancement. From this study alone, the tamarins show perception similar to those of people with autism to sparse displays, but with particular kinds of training with shapes that emphasize the global property, individuals of this species could overcome their fixation on processing at both local and global levels and show a global advantage. It is an intriguing endeavor to develop training programs that would induce monkeys to see the forest despite the details of the trees and to determine whether cognitive training would induce hierarchical assessments in individuals with autism as well.

\section{References}

Brock, J., Brown, C., Boucher, J., \& Ripon, G. (2002). The temporal binding deficit hypothesis of autism. Development and Psychopathology, 14, 209-224.

Brosnan, M. J., Scott, F. J., Fox, S., \& Pye, J. (2004). Gestalt processing in autism: Failure to process perceptual relationships and the implications for contextual understanding. Journal of Child Psychology and Psychiatry, 45, 459-469.

Cassia, V. M., Simion, F., Milani, E., \& Umilta, C. (2002). Dominance of global visual properties at birth. Journal of Experimental Psychology: General, 131, 398-411.

Cohen, J. D., MacWhinney, B., Flatt, M., \& Provost, J. (1993). PsyScope: A new graphic interactive environment for designing psychology experiments. Behavioral Research Methods, Instruments \& Computers, 25, 257-271.

De Lillo, C., Spinozzi, G., Truppa, V., \& Naylor, D. M. (2005). A comparative analysis of global and local processing of hierarchical visual stimuli in young children (Homo sapiens) and monkeys (Cebus apella). Journal of Comparative Psychology, 119, 155-165.

De Valois, R. L., \& Jacobs, G. H. (1968, November). Primate color vision. Science, 16, 533-540.

Dukette, D., \& Stiles, J. (1996). Children's analysis of hierarchical patterns: Evidence from a similarity judgment task. Journal of Experimental Child Psychology, 63, 103-140.

Dukette, D., \& Stiles, J. (2001). The effects of stimulus density on chil- 
dren's analysis of hierarchical patterns. Developmental Science, 4, 233251

Fagot, J., \& Deruelle, C. (1997). Processing of global and local visual information and hemispherical specialization in humans (Homo sapiens) and baboons (Papio papio). Journal of Experimental Psychology: Human Perception and Performance, 23, 429-442.

Fagot, J., \& Tomonaga, M. (1999). Global and local processing in humans (Homo sapiens) and chimpanzees (Pan troglodytes): Use of a visual search task with compound stimuli. Journal of Comparative Psychology, $113,3-12$.

Fagot, J., Tomonaga, M., \& Deruelle, C. (2001). Processing of the global and local dimensions of visual hierarchical stimuli by humans (Homo sapiens), chimpanzees (Pan troglodytes), and baboons (Papio papio). In Matsuzawa, T. (Ed.). Primate origins of human cognition and behavior (pp. 87-103). New York: Springer-Verlag.

Fobes, J. L., \& King, J. E. (1982). Vision: The dominant primate modality. In Fobes, J. L., \& King, L. E. (Eds.), Primate behavior (219-243). New York: Academic Press.

Fremouw, T., Herbranson, W. T., \& Shimp, C. P. (2002). Dynamic shifts of pigeon local/global attention. Animal Cognition, 5, 233-243.

Fujita, K., \& Matsuzawa, T. (1990). Delayed figure reconstruction by a chimpanzee (Pan troglodytes), and humans (Homo sapiens). Journal of Comparative Psychology, 104, 345-351.

Ghim, H. R., \& Eimas, P. D. (1988). Global and local processing by 3- and 4-month-old infants. Perception \& Psychophysics, 43, 165-171.

Hopkins, W. D., \& Washburn, D. A. (2002). Matching visual stimuli on the basis of global and local features by chimpanzees (Pan troglodytes) and rhesus monkeys (Macaca mulatta). Animal Cognition, 5, 27-31.

Joliffe, T., \& Baron-Cohen, S. (1997). Are people with autism and Asperger's syndrome faster than normal on the embedded figures test? Journal of Child Psychology and Psychiatry, 38, 527-534.

Kimchi, R. (1990). Children's perceptual organization of hierarchical patterns. European Journal of Cognitive Psychology, 2, 133-149.

Kimchi, R. (1992). Primacy of holistic processing and global/local paradigm: A critical review. Psychological Bulletin, 112, 24-38.

Mottron, L., Belleville, S., \& Menard, E. (1999). Local bias in autistic subjects as evidenced by graphic tasks: Perceptual hierarchization or working memory deficit? Journal of Child Psychology and Psychiatry, 40, 743-755.

Navon, D. (1977). Forest before trees: The precedence of global features in visual perception. Cognitive Psychology, 9, 353-383.

Navon, D. (1981). The forest revisited: More on global precedence. Psychological Research, 43, 1-32.

Neiworth, J. J., Anders, S. L., \& Parsons, R. R. (2001). Tracking responses related to self-recognition: A frequency comparison of responses to mirrors, photographs, and videotape of monkeys by cotton top tamarins (Saguinus oedipus). Journal of Comparative Psychology, 115, 432-438.

Neiworth, J. J., Burman, M. A., Basile, B. M., \& Lickteig, M. T. (2002). Use of experimenter-given cues in visual co-orienting and in an objectchoice task by a new world monkey species, cotton top tamarins (Saguinus oedipus). Journal of Comparative Psychology, 116, 3-11.

Neiworth, J. J., Parsons, R. R., \& Hassett, J. M. (2004). A test of the generality of perceptually-based categories found in infants: Attentional differences toward natural kinds by New World monkeys. Developmental Science, 7, 185-193.

Neiworth, J. J., Steinmark, E., Basile, B. M., Wonders, R., Steely, F., \& DeHart, C. (2003). A test of object permanence in a New World monkey species, cotton top tamarins (Saguinus oedipus). Animal Cognition, 6, 27-37.

Quinlan, P., \& Wilton, R. (1998). Grouping by proximity or similarity? Competition between the gestalt principles in vision. Perception, 27, 417-430.

Quinn, P. C., Bhatt, R. S., Brush, D., Grimes, A., \& Sharpnack, H. (2002). Development of form similarity as a Gestalt grouping principle in infancy. Psychological Science, 4, 320-328.

Shah, A., \& Frith, U. (1983). An islet in ability of autistic children: A research note. Journal of Child Psychology and Psychiatry, 24, 613620 .

Spinozzi, G., De Lillo, C., \& Truppa, V. (2003). Global and local processing of hierarchical visual stimuli in tufted capuchin monkeys (Cebus apella). Journal of Comparative Psychology, 117, 15-23.

Tanaka, H., \& Fujita, I. (2000, September 11). Global and local processing of visual patterns in macaques monkeys. Neuroreport: For Rapid Communication of Neuroscience Research, 11, 2881-2884.

Tomonaga, M., \& Matsuzawa, T. (1992). Perception of complex geometric figures in chimpanzees (Pan troglodytes) and humans (Homo sapiens): Analyses of visual similarity on the basis of choice reaction time. Journal of Comparative Psychology, 106, 43-52.

Triesman, A., \& Peterson, R. (1984). Emergent features, attention and object perception. Journal of Experimental Psychology: Human Perception and Performance, 10, 12-31.

van Kleek, M., \& Kosslyn, S. (1989). Gestalt laws of perceptual organization in an embedded figures task: Evidence for hemispheric specialization. Neuropsychologia, 27, 1179-1186.

Received December 31, 2005

Revision received April 6, 2006

Accepted April 7, 2006 